\title{
Classical Trajectory Study of Mode Specificity and Rotational Effects in Unimolecular Dissociation of $\mathrm{HO}_{2}$
}

\author{
J. M. C. Marques and A. J. C. Varandas* \\ Departamento de Química, Universidade de Coimbra, P-3049 Coimbra Codex, Portugal
}

Received: March 17, 1997; In Final Form: May 7, $1997^{\otimes}$

\begin{abstract}
Trajectory calculations are presented for the unimolecular dissociation of $\mathrm{HO}_{2}$. The study covers internal energies in the range $58.311 \leq E_{\text {tot }} / \mathrm{kcal} \mathrm{mol}^{-1} \leq 59.432$, just above the $\mathrm{H}+\mathrm{O}_{2}$ threshold, and $E_{\text {tot }}=76.412$ $\mathrm{kcal} \mathrm{mol}^{-1}$ for which the $\mathrm{O}+\mathrm{OH}$ channel is also open. The $\mathrm{HO}_{2}$ single-valued double many-body expansion potential energy surface has been employed in all calculations. Due to strong coupling among the vibrational degrees of freedom, mode specificity is shown to play a minor role in the formation of $\mathrm{H}+\mathrm{O}_{2}$. Conversely, the increase of initial rotational energy clearly influences the dynamics of the unimolecular dissociation. In particular, energy placed in a specific rotational degree of freedom can dramatically modify the yield of $\mathrm{O}_{2}$ or $\mathrm{OH}$ products and corresponding decay rates. The results show the importance of rotational effects in order to correctly describe the unimolecular dissociation of $\mathrm{HO}_{2}$.
\end{abstract}

\section{Introduction}

Unimolecular systems are usually employed in the study of energy transfer phenomena among internal degrees of freedom. In this context, most published theoretical work has focused on topics such as intramolecular vibrational energy redistribution, isomerization between two minima of the potential surface, nonlinear processes and chaos, and unimolecular dissociation. Since the number of degrees of freedom increases with the number of atoms in the molecule, tetratomic and larger molecules are usually employed in such studies. However, having only three (four) vibrational modes, nonlinear (linear) triatomic molecules can provide a more clear understanding of unimolecular processes (e.g., refs 1 and 2 and references therein).

The Rampsberger-Rice-Kassel-Marcus (RRKM) theory ${ }^{3,4}$ has been successfully applied in the study of a wide variety of unimolecular reactions. This statistical theory is based on the assumption that intramolecular energy redistribution is rapid in comparison with the reaction time. Thus, non-RRKM behavior is associated with the fact that modes relax at different rates, and coupling between different degrees of freedom depends both on its nature and level of excitation, which may originate nonlinear resonances. In other words, intramolecular energy transfer occurs via specific pathways. A well-studied situation favoring rate enhancement by specific mode excitation is the reaction over a low potential barrier, which has been explored by Thompson and co-workers ${ }^{5,6}$ in their studies of HONO cistrans isomerization. The HONO molecule has also been used to investigate the influence of rotation and angular momentum orientation in the rate of dissociation. ${ }^{7}$ More recently, Marks ${ }^{8}$ has searched for mode-specific effects in the isomerization of $\mathrm{CD}_{2} \mathrm{HNC}$ and found that rotational excitation about the molecular axis enhances the corresponding rates.

Rotational effects on the unimolecular dissociation of the $\mathrm{HO}_{2}$ system have been previously reported by Miller and Brown ${ }^{9}$ using the Melius - Blint ${ }^{10}$ potential energy surface, which shows an unphysical barrier for dissociation into $\mathrm{H}+\mathrm{O}_{2}$. However, it has been the emergence of a realistic single-valued double many-body expansion ${ }^{11}$ potential surface for $\mathrm{HO}_{2}$ (known as DMBE IV) that induced considerable theoretical interest during

${ }^{\otimes}$ Abstract published in Advance ACS Abstracts, June 15, 1997. the past few years on the dissociation reaction

$$
\mathrm{HO}_{2}^{*} \rightarrow \mathrm{O}_{2}+\mathrm{H}
$$

The star in $\mathrm{HO}_{2}^{*}$ implies an energized species. Recently, Mandelshtam et al. ${ }^{12}$ have reported a detailed investigation of bound states and resonances for the nonrotating $(\mathbf{J}=0)$ hydroperoxyl radical up to an energy just above the $\mathrm{H}+\mathrm{O}_{2}$ dissociation threshold and concluded that the $\mathrm{HO}_{2}$ system is dominantly irregular, especially at high energies close to the dissociation threshold. Motivated by this work, Mahapatra ${ }^{13}$ has shown that the time domain behavior of the bound states and resonances of the $\mathrm{HO}_{2}$ radical is extremely chaotic, which is in excellent agreement with the corresponding Wigner nearestneighbor spacing distributions reported by Mandelshtam et al. ${ }^{12}$ and by Dobbyn et al. ${ }^{14}$ Moreover, these authors ${ }^{15}$ have concluded that the $\mathrm{HO}_{2}$ system is classically chaotic (at energies near the $\mathrm{H}+\mathrm{O}_{2}$ threshold), presenting strong fluctuations on the resonance widths and random lifetime distributions of the classical trajectories. However, Dobbyn et al. ${ }^{14}$ have also found that, although the wave functions are mostly irregular around the $\mathrm{H}+\mathrm{O}_{2}$ dissociation threshold, there are some regular states that are associated with extensive excitation in the OO stretching mode. Indeed, using quantum and semiclassical variational transition state models, Song et al. ${ }^{16}$ have calculated microcanonical rate constants for reaction 1 , and concluded from a comparison of the different results that there is weak coupling between the $\mathrm{OO}$ stretching and $\mathrm{OOH}$ bending modes along the reaction path. Moreover, we have recently found ${ }^{17}$ that dissociation of energized $\mathrm{HO}_{2}^{*}$ complexes arising from $\mathrm{H}+\mathrm{ArO}_{2}$ collisions follows essentially a non-RRKM behavior, which could apparently be due to vibrational mode specificity.

In this work, we report a classical dynamics study of the unimolecular dissociation for internal energies in which reaction 1 is the only open channel and also for formation of both $\mathrm{H}+$ $\mathrm{O}_{2}$ and $\mathrm{O}+\mathrm{OH}$ products. Our main goals are to study the effect of rotation in $\mathrm{HO}_{2}^{*}$ unimolecular dissociation and search for vibrational mode specificity in this reactive process. Thus, after a brief presentation of the methodology in section 2, the results are reported and discussed in section 3. Section 4 summarizes the conclusions. 
TABLE 1: Results of the Trajectory Calculations for the Nonrotating HO* Molecule ${ }^{a}$

\begin{tabular}{|c|c|c|c|c|c|c|}
\hline \multirow[b]{2}{*}{$E_{\text {tot }} / \mathrm{kcal} \mathrm{mol}^{-1}$} & \multirow[b]{2}{*}{$\left(v_{1}, v_{2}, \nu_{3}\right)$} & \multirow[b]{2}{*}{$N_{\text {isom }}^{b}$} & \multicolumn{4}{|c|}{$\mathrm{H}+\mathrm{O}_{2}$ products } \\
\hline & & & $f_{\langle E v\rangle}$ & $f_{\langle E \underline{r}\rangle}$ & $f_{\langle E \mathrm{Er}\rangle}$ & $k_{\mathrm{b}} / \mathrm{ps}^{-1}$ \\
\hline 59.432 & $c$ & $1985(3)$ & 0.37 & 0.04 & 0.59 & 0.91 \\
\hline 59.432 & $(3,3,3)$ & $1970(3)$ & 0.39 & 0.04 & 0.57 & 0.92 \\
\hline 58.857 & $(16,0,0)$ & $2400(4)$ & 0.36 & 0.04 & 0.60 & 0.68 \\
\hline 58.780 & $(0,13,0)$ & 2744(5) & 0.37 & 0.04 & 0.59 & 0.68 \\
\hline 58.311 & $(0,0,5)$ & 2353(4) & 0.38 & 0.04 & 0.58 & 0.69 \\
\hline
\end{tabular}

${ }^{a}$ All energies are above $\mathrm{H}+\mathrm{O}_{2}$ and below $\mathrm{O}+\mathrm{OH}$ dissociation thresholds. ${ }^{b}$ Total number of isomerizations for trajectories forming $\mathrm{H}+\mathrm{O}_{2}$. ${ }^{c}$ Initial conditions chosen using a microcanonical sampling procedure.

\section{Method}

All calculations reported in the present work have employed the DMBE IV ${ }^{11}$ potential energy surface for the electronic ground state of the $\mathrm{HO}_{2}$ radical. For the purpose of the present calculations, its most relevant features are the two deep equivalent wells associated with the corresponding $\mathrm{HO}_{2}$ isomers, which are separated by a saddle point structure. Since the DMBE IV potential energy surface has been extensively used for dynamics studies ${ }^{11,17-26}$ involving the $\mathrm{HO}_{2}$ radical, we omit here any further details.

The classical trajectory method has been employed to calculate the unimolecular decay rates for reaction 1. All calculations have been done using an adapted version of the MERCURY $^{27}$ computer code to run batches of typically 500 trajectories for various selected initial vibrational and rotational energies. After proper optimization the integration step has been fixed at $3.0 \times 10^{-17} \mathrm{~s}$. Trajectories are considered to be reactive whenever the forming products are separated by more than 5 $\AA$. Nondissociative trajectories are allowed to last up to $60 \mathrm{ps}$.

To define the initial conditions, we have assigned vibrational quanta $\left(v_{1}, v_{2}, v_{3}\right)$ to each normal mode with random vibrational phases $^{28,29}$ or, alternatively, used a microcanonical sampling method to distribute the vibrational energy among the three modes. Thus, the vibrational energy is given by the sum of the three normal mode energies

$$
E_{\mathrm{v}}=E_{\mathrm{OO}}+E_{\mathrm{OOH}}+E_{\mathrm{OH}}
$$

being the fundamental frequencies 1101,1353 , and $3485 \mathrm{~cm}^{-1}$ for $\mathrm{OO}$ stretching, $\mathrm{OOH}$ bending, and $\mathrm{OH}$ stretching, respectively. Once the normal mode energies have been assigned, the nonseparable Hamiltonian of the system is calculated and the Cartesian coordinates and momenta are scaled to give the required vibrational energy ${ }^{30}$ within a tolerence of $\sim 10^{-3} \mathrm{kcal}$ $\mathrm{mol}^{-1}$ or so. During the scaling procedure, spurious linear and angular momenta created in the molecule by the vibrational excitation are removed.

The rotational energy assignment has been done by calculating the magnitude of the angular momentum vector corresponding to the specified rotational energy, $E_{\mathrm{r}}$. Since the vibrational angular momentum has already been removed, one has

$$
E_{\mathrm{r}}=\frac{1}{2} \mathbf{L}^{\mathrm{T}} \mathbf{I}^{-1} \mathbf{L}
$$

where $\mathbf{L}^{\mathrm{T}}$ is the transpose of the angular momentum vector and $\mathbf{I}^{-1}$ is the inverse of the momentum of inertia tensor, which is calculated for every specific initial geometry of the molecule after the assignment of the vibrational energy. Note that the axis of rotation is defined by the orientation of the total angular momentum, and hence it is established by providing the corresponding direction cosine vector $\mathbf{D}$. Thus, using eq 3 , the magnitude of the total angular momentum is given by

$$
|\mathbf{L}|=\left(\frac{2 E_{\mathrm{r}}}{\mathbf{D}^{\mathrm{T}} \mathbf{I}^{-1} \mathbf{D}}\right)^{1 / 2}
$$

where it is implicit that each component of $\mathbf{L}$ can be written as

$$
L_{\mathrm{i}}=|\mathbf{L}| D_{\mathrm{i}}
$$

Each of the three direction cosine components $\left(D_{\mathrm{i}}\right)$ may then be specified as 0 or 1 , according to the selected orientation for the $\mathbf{L}$ vector, and multiplied by the appropriate $1 /\left(\sum_{\mathrm{i}} D_{\mathrm{i}}\right)^{1 / 2}$ normalizing factor. Finally, the angular velocity can be written in matrix form as

$$
\omega=\mathbf{I}^{-1} \mathbf{L}
$$

and the velocity $\omega \times \mathbf{R}_{i}$ due to rotation added to each atom; $\mathbf{R}_{i}$ is the position vector of the $i$ th atom.

After the trajectories have been calculated for different sets of initial conditions, it is possible to characterize the dissociation behavior of $\mathrm{HO}_{2}^{*}$ and obtain the corresponding rates. To describe non-RRKM behavior in the decaying molecules, we have considered the following two-step mechanism ${ }^{17}$

$$
\mathrm{A} \stackrel{k_{\mathrm{a}}}{\longrightarrow} \mathrm{B} \stackrel{k_{\mathrm{b}}}{\longrightarrow} \text { products }
$$

where A (B) stands for $\mathrm{HO}_{2}^{*}$ molecules not coupled (strongly coupled) with the reaction path and the rate coefficients follow the relation $k_{\mathrm{a}}<k_{\mathrm{b}}$ or $k_{\mathrm{a}} \ll k_{\mathrm{b}}$. Using such a scheme, the calculated fraction of $\mathrm{HO}_{2}^{*}$ molecules present at each moment can be fitted to the form ${ }^{17}$

$$
\begin{aligned}
f(t)=f_{\mathrm{A}}+f_{\mathrm{B}}=\left(f_{\mathrm{B}}^{0}-\frac{k_{\mathrm{a}} f_{\mathrm{A}}^{0}}{k_{\mathrm{b}}-k_{\mathrm{a}}}\right) & \exp \left(-k_{\mathrm{b}} t\right)+ \\
& \left(\frac{k_{\mathrm{a}} f_{\mathrm{A}}^{0}}{k_{\mathrm{b}}-k_{\mathrm{a}}}+f_{\mathrm{A}}^{0}\right) \exp \left(-k_{\mathrm{a}} t\right)
\end{aligned}
$$

where $f_{\mathrm{A}}$ and $f_{\mathrm{B}}$ are the fractions of $\mathrm{A}$ and $\mathrm{B}$ at time $t$ and $f_{\mathrm{A}}^{0}$ and $f_{\mathrm{B}}^{0}$ are the corresponding values for $t=0 ; f_{\mathrm{A}}^{0}, f_{\mathrm{B}}^{0}, k_{\mathrm{a}}$, and $k_{\mathrm{b}}$ are adjustable parameters. Thus, if the kinetics is essentially RRKM type, it can be described using

$$
f(t)=f_{\mathrm{B}}^{0} \exp \left(-k_{\mathrm{b}} t\right)
$$

where it has been assumed that all $\mathrm{HO}_{2}^{*}$ molecules are B-type (i.e., $f_{\mathrm{A}}^{0}=0$ ); see also ref 17 .

\section{Results and Discussion}

Mode specificity in $\mathrm{HO}_{2}^{*}$ dissociation has been investigated for the nonrotating system with internal energies varying from 58.311 to $59.432 \mathrm{kcal} \mathrm{mol}^{-1}$. The results are presented in Table 

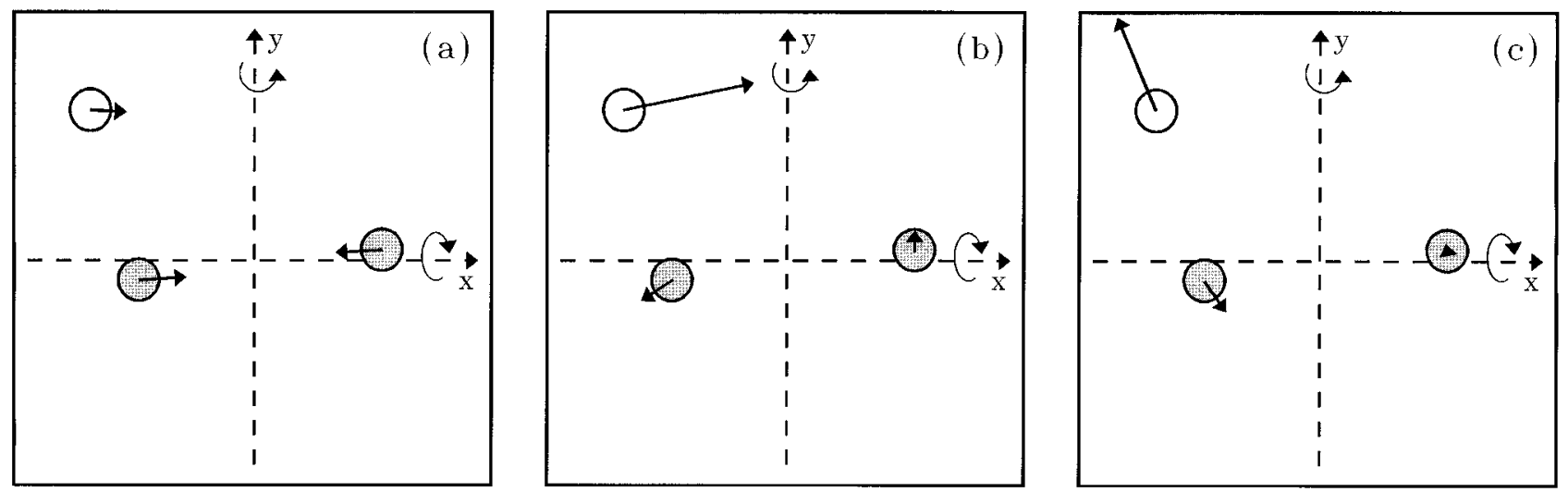

Figure 1. Schematic representation of the three normal modes of vibration for $\mathrm{HO}_{2}$ shown in the system of coordinates associated to the principal axis of inertia: (a) $\mathrm{OO}$ stretch, (b) $\mathrm{OOH}$ bend, and (c) $\mathrm{OH}$ stretch. Note that the $x$-axis and $y$-axis are represented by dashed lines, while the $z$-axis is perpendicular to the plane of the figure. For clarity, the oxygen atoms are shown by the shadowed circles, and the hydrogen atom is represented by the open one.

1 and comprise the number of $\mathrm{HO}_{\mathrm{a}} \mathrm{O}_{\mathrm{b}} \rightarrow \mathrm{HO}_{\mathrm{b}} \mathrm{O}_{\mathrm{a}}(a$ and $b$ label the $\mathrm{O}$ atoms) isomerizations, the average fractions of vibrational, rotational, and translational energies in the products, and the values of the dissociation rate coefficients $k_{\mathrm{b}}$. Four different sets of initial vibrational quantum numbers $\left(v_{1}, v_{2}, v_{3}\right)$ have been investigated, in addition to a microcanonical distribution of the vibrational energy. Table 1 shows that the total number of isomerizations (the average number of isomerizations per trajectory is given in parentheses) increases with excitation of the bending mode; for a pictorial representation of the normal mode eigenvectores, see Figure 1. Moreover, we observe a decrease in the total number of isomerizations when passing from the $(16,0,0)$ to the $(0,0,5)$ excitation, as might be anticipated from the inspection of the corresponding eigenvectors for the $\mathrm{OO}$ (Figure 1a) and $\mathrm{OH}$ (Figure 1c) stretching modes. In addition, there is a decrease in the number of isomerizations (the average value per trajectory is now reduced to three) for the sets of initial conditions comprising the $(3,3,3)$ excitation and microcanonical normal mode sampling. Indeed, as shown in Table 1, there is an almost coincidence between these two sets of results.

Particularly interesting from Table 1 are the fractions of vibrational, rotational, and translational energies in the product $\mathrm{O}_{2}$ molecules. They are $\sim 37 \%$ for vibration, $4 \%$ for rotation, and $\sim 59 \%$ for translation, these values being kept approximately invariant with respect to the initial excitation. This suggests that all vibrational modes are strongly coupled, which allows a fast energy redistribution in the $\mathrm{HO}_{2}^{*}$ complex and hence a RRKM-type behavior for the decay rates of the unimolecular reaction in eq 1. In fact, Figure 2 shows that the trajectory results are well fitted by a simple inverse exponential form of eq 9. This is in good agreement with recent classical and quantum calculations for $\mathbf{J}=0 .{ }^{15}$ It is also seen from this figure (panel a) that the results are nearly coincident for the one-mode excitations $(16,0,0),(0,13,0)$, and $(0,0,5)$, although some scattering is present for the longest dissociation times. A similar finding is observed from panel $\mathrm{b}$ for the $(3,3,3)$ and microcanonical vibrational excitations. However, we note that the unimolecular dissociation rate coefficients are higher in this case than those corresponding to the three one-mode excitations as could be seen by plotting the curves for $k_{\mathrm{b}}=0.91$ and 0.68 $\mathrm{ps}^{-1}$ on the same plot (see also Table 1). We are therefore led to conclude that the coupling among "democratically" excited vibrational modes is more efficient to give dissociative outcomes. Indeed, for the $(16,0,0),(0,13,0)$, and $(0,0,5)$ excitations the decay rates decrease to about $0.7 \mathrm{ps}^{-1}$. Although not very significant, the small increase in $k_{\mathrm{b}}$ for the $(0,0,5)$ case may be

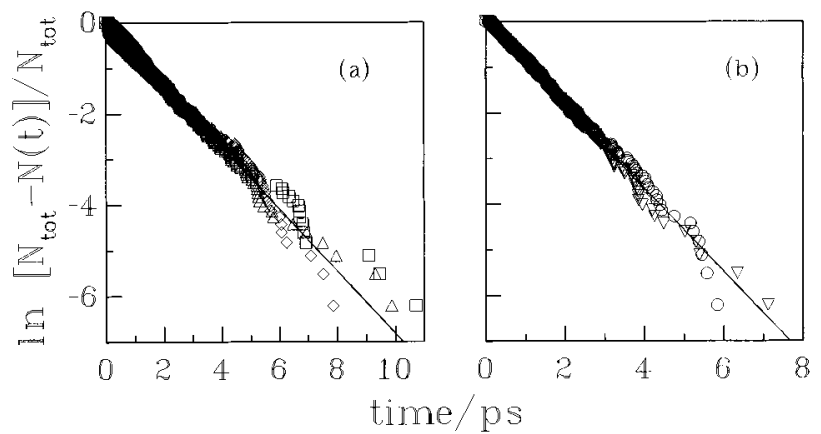

Figure 2. Logarithm of the decay rate for nonrotating $\mathrm{HO}_{2}^{*}$ molecules as a function of time. Panel a is for specific normal mode excitations: $\diamond,(16,0,0) ; \square,(0,13,0) ; \Delta,(0,0,5)$. Panel b compares the logarithms of the decay rates for a microcanonical vibrational energy distribution $(O)$ and the $(3,3,3)$ excitation $(\nabla)$, both corresponding to the initial vibrational energy $E_{\mathrm{v}}=59.432 \mathrm{kcal} \mathrm{mol}^{-1}$. The lines represent the least-squares fits of the points to eq 9; only one fit is shown since they are indistinguishable within the scale of the figure for the different sets of points.

an indication that the $\mathrm{OH}$ stretch (Figure 1c) is better coupled to the reaction coordinate than the other modes, as already been advanced elsewhere. ${ }^{14,17}$

We now address the influence of rotation in the $\mathrm{HO}_{2}^{*}$ unimolecular dissociation, the results being gathered in Tables 2 and 3 for $\mathrm{H}+\mathrm{O}_{2}$ formation and in Table 4 for dissociation leading to $\mathrm{O}+\mathrm{OH}$ products. Table 2 summarizes the results obtained for total internal energy $E_{\mathrm{tot}}=59.432 \mathrm{kcal} \mathrm{mol}^{-1}$, which is only $4.569 \mathrm{kcal} \mathrm{mol}^{-1}$ above the classical threshold for the $\mathrm{H}+\mathrm{O}_{2}$ dissociative channel and significantly below the $\mathrm{O}+\mathrm{OH}$ one $\left(\sim 68 \mathrm{kcal} \mathrm{mol}^{-1}\right)$. It is seen from column three of this table that the number of isomerizations increases with increasing rotational energy. This is not unexpected since rotation is more strongly coupled with the bending mode. Also, the fraction of rotational energy in the $\mathrm{H}+\mathrm{O}_{2}$ products increases from 0.04 for the nonrotating $\mathrm{HO}_{2}^{*}$ to 0.47 for $E_{\mathrm{r}}=33.961 \mathrm{kcal}$ $\mathrm{mol}^{-1}$, which suggests that the initial rotational energy appears in the products preferentially as rotation. In contrast, $f_{\left\langle E^{\prime}\right\rangle}$ decreases with the increase of $\mathrm{HO}_{2}^{*}$ rotational energy, which suggests that most of the vibrational energy in the products comes from the $\mathrm{HO}_{2}^{*}$ vibrational degrees of freedom. Note also that the fraction of the $\mathrm{H}+\mathrm{O}_{2}$ translational energy decreases, in a nearly proportional way, as the rotational (vibrational) energy increases (decreases) in $\mathrm{HO}_{2}^{*}$. In turn, it appears that most of the product's translational energy is provided directly from the initial vibrational degrees of freedom. 
TABLE 2: Trajectory Results for Total Internal Energy $E_{\text {tot }}=59.432 \mathrm{kcal} \mathrm{mol}^{-1} a$

\begin{tabular}{|c|c|c|c|c|c|c|c|}
\hline \multicolumn{2}{|c|}{ initial conditions } & \multirow[b]{2}{*}{$N_{\text {isom }}^{b}$} & \multicolumn{5}{|c|}{$\mathrm{H}+\mathrm{O}_{2}$ products } \\
\hline$E_{\mathrm{r}} / \mathrm{kcal} \mathrm{mol}^{-1}$ & $(x y z)$ & & $f_{\langle E v\rangle}$ & $f_{\langle E \hat{r}\rangle}$ & $f_{\langle E \mathrm{tr}\rangle}$ & $k_{\mathrm{a}} / \mathrm{ps}^{-1}$ & $k_{\mathrm{b}} / \mathrm{ps}^{-1}$ \\
\hline \multirow[t]{4}{*}{6.816} & (111) & $2285(4)$ & 0.28 & 0.16 & 0.56 & & 0.51 \\
\hline & (100) & $1908(3)$ & 0.31 & 0.08 & 0.61 & & 0.64 \\
\hline & (010) & $3674(40)$ & 0.07 & 0.53 & 0.40 & $0.002^{c}$ & $0.073^{d}$ \\
\hline & (001) & $986(30)$ & 0.03 & 0.50 & 0.47 & $0.0002^{e}$ & $0.11^{f}$ \\
\hline 16.980 & (111) & $4657(9)$ & 0.19 & 0.30 & 0.51 & $0.057^{g}$ & $0.23^{h}$ \\
\hline 33.961 & (111) & $8115(26)$ & 0.11 & 0.47 & 0.42 & $0.013^{i}$ & $0.10^{j}$ \\
\hline
\end{tabular}

${ }^{a}$ Initial vibrational mode energies have been chosen using a microcanonical sampling procedure. ${ }^{b}$ Total number of isomerizations for trajectories forming $\mathrm{H}+\mathrm{O}_{2} \cdot{ }^{c} f_{\mathrm{A}}^{0}=0.90 .{ }^{d} f_{\mathrm{B}}^{0}=0.10 .{ }^{e} f_{\mathrm{A}}^{0}=0.95 \cdot{ }^{f} f_{\mathrm{B}}^{0}=0.05 \cdot{ }^{g} f_{\mathrm{A}}^{0}=0.21 \cdot{ }^{h} f_{\mathrm{B}}^{0}=0.79 \cdot{ }^{i} f_{\mathrm{A}}^{0}=0.72 .{ }^{j} f_{\mathrm{B}}^{0}=0.28$.

TABLE 3: Trajectory Results for Formation of $\mathrm{H}+\mathrm{O}_{2}$ Products at Total Internal Energy $E_{\text {tot }}=76.412 \mathrm{kcal}^{\mathrm{mol}}{ }^{-1}$

\begin{tabular}{|c|c|c|c|c|c|c|c|c|}
\hline \multicolumn{2}{|c|}{ initial conditions } & \multirow[b]{2}{*}{$N_{\text {isom }}^{a}$} & \multicolumn{6}{|c|}{$\mathrm{H}+\mathrm{O}_{2}$ products } \\
\hline$E_{\mathrm{r}} / \mathrm{kcal} \mathrm{mol}^{-1}$ & $(x y z)$ & & $N_{\mathrm{r}}$ & $f_{\left\langle E^{\prime}\right\rangle}$ & $f_{\left\langle E^{\prime}\right\rangle}$ & $f_{\langle E \mathrm{rr}\rangle}$ & $k_{\mathrm{a}} / \mathrm{ps}^{-1}$ & $k_{\mathrm{b}} / \mathrm{ps}^{-1}$ \\
\hline 0.0 & & $540(1)$ & 369 & 0.34 & 0.04 & 0.62 & & 5.47 \\
\hline \multirow[t]{4}{*}{16.980} & (111) & $532(1)$ & 497 & 0.27 & 0.11 & 0.62 & & 4.04 \\
\hline & (100) & $505(1)$ & 496 & 0.28 & 0.06 & 0.66 & & 4.34 \\
\hline & (010) & $1086(3)$ & 359 & 0.12 & 0.51 & 0.37 & $0.30^{b}$ & $1.34^{c}$ \\
\hline & (001) & $895(3)$ & 290 & 0.11 & 0.47 & 0.42 & $0.26^{d}$ & $1.47^{e}$ \\
\hline 23.797 & (111) & $480(<1)$ & 495 & 0.24 & 0.14 & 0.62 & & 3.51 \\
\hline
\end{tabular}

${ }^{a}$ Total number of isomerizations for trajectories forming $\mathrm{H}+\mathrm{O}_{2} \cdot{ }^{b} f_{\mathrm{A}}^{0}=0.11 .{ }^{c} f_{\mathrm{B}}^{0}=0.89 .{ }^{d} f_{\mathrm{A}}^{0}=0.05 .{ }^{e} f_{\mathrm{B}}^{0}=0.95$.

TABLE 4: Trajectory Results for Formation of $O+O H$ Products at Total Internal Energy $E_{\text {tot }}=76.412 \mathrm{kcal} \mathrm{mol}^{-1}$

\begin{tabular}{|c|c|c|c|c|c|c|c|c|}
\hline \multicolumn{2}{|c|}{ initial conditions } & \multirow[b]{2}{*}{$N_{\text {isom }}^{a}$} & \multicolumn{6}{|c|}{$\mathrm{O}+\mathrm{OH}$ products } \\
\hline$E_{\mathrm{r}} / \mathrm{kcal} \mathrm{mol}^{-1}$ & $(x y z)$ & & $N_{\mathrm{r}}$ & $f_{\left\langle E^{\prime}\right\rangle}$ & $f_{\langle E \hat{r}\rangle}$ & $f_{\langle E t r\rangle}$ & $k_{\mathrm{a}} / \mathrm{ps}^{-1}$ & $k_{\mathrm{b}} / \mathrm{ps}^{-1}$ \\
\hline 0.0 & & $90(<1)$ & 131 & 0.43 & 0.17 & 0.40 & & 3.22 \\
\hline 16.980 & (111) & $8(2)$ & 3 & $b$ & $b$ & $b$ & $b$ & $b$ \\
\hline & (100) & $12(3)$ & 4 & $b$ & $b$ & $b$ & $b$ & $b$ \\
\hline & $(010)$ & $903(6)$ & 138 & 0.22 & 0.19 & 0.59 & $0.12^{c}$ & $1.36^{d}$ \\
\hline & (001) & $1137(5)$ & 210 & 0.21 & 0.12 & 0.67 & $0.06^{e}$ & $1.10^{f}$ \\
\hline 23.797 & (111) & $8(1)$ & 5 & $b$ & $b$ & $b$ & $b$ & $b$ \\
\hline
\end{tabular}

${ }^{a}$ Total number of isomerizations for trajectories forming $\mathrm{O}+\mathrm{OH} .{ }^{b}$ The number of trajectories forming $\mathrm{O}+\mathrm{OH}$ is too low to obtain reliable statistics. ${ }^{c} f_{\mathrm{A}}^{0}=0.32 .{ }^{d} f_{\mathrm{B}}^{0}=0.68 .{ }^{e} f_{\mathrm{A}}^{0}=0.13 .{ }^{f} f_{\mathrm{B}}^{0}=0.87$.

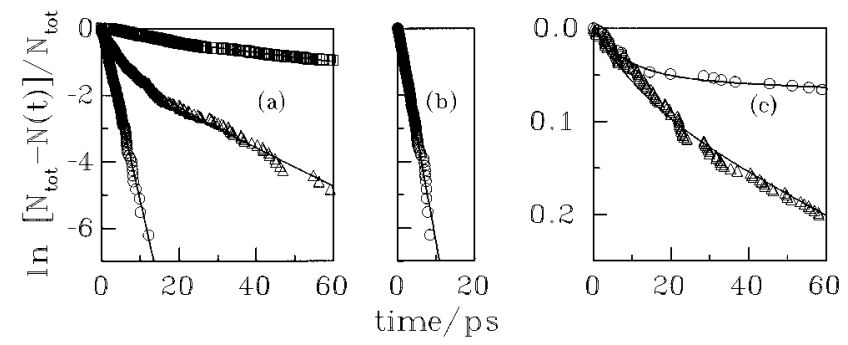

Figure 3. Logarithm of the decay rate for rotating $\mathrm{HO}_{2}^{*}$ molecules as a function of time at $E_{\mathrm{tot}}=59.432 \mathrm{kcal} \mathrm{mol}^{-1}$. Panel a: $\bigcirc, E_{\mathrm{r}}=6.816$ $\mathrm{kcal} \mathrm{mol}^{-1} ; \Delta, E_{\mathrm{r}}=16.980 \mathrm{kcal} \mathrm{mol}^{-1} ; \square, E_{\mathrm{r}}=33.961 \mathrm{kcal} \mathrm{mol}^{-1}$. Panel b: $O, E_{\mathrm{r}}=6.816 \mathrm{kcal} \mathrm{mol}^{-1}$ and (100) excitation. Panel c: $\Delta$, $E_{\mathrm{r}}=6.816 \mathrm{kcal} \mathrm{mol}^{-1}$ with rotational energy associated to $L_{y} ; \bigcirc, L_{z}$. See text.

Figure 3a shows the logarithm of the decay rates as a function of time for different values of the rotational excitation. It is clear from this figure and Tables 1 and 2 that $k_{\mathrm{b}}$ decreases (from 0.91 to $0.51 \mathrm{ps}^{-1}$ ) as the rotational energy increases (from zero to $6.816 \mathrm{kcal} \mathrm{mol}^{-1}$, respectively), while continuing to decrease for even higher rotational energies. This is in accordance with the resulting increase in the centrifugal barrier. Although some decrease in the value of the dissociation rate coefficients should arise, the system would be expected to follow the same exponential behavior described by eq 9 . This is the case shown in Figures $2 \mathrm{~b}$ and Figure $3 \mathrm{a}$ for the nonrotating and $E_{\mathrm{r}}=6.816$ $\mathrm{kcal} \mathrm{mol}^{-1}$ cases, respectively. However, as the rotational excitation increases for $E_{\mathrm{r}}=16.980 \mathrm{kcal} \mathrm{mol}^{-1}$ and $E_{\mathrm{r}}=33.961$ $\mathrm{kcal} \mathrm{mol}^{-1}$, the system does not present a single-exponential behavior, and the two-step mechanism ${ }^{17}$ described in section 2 (see eqs 7 and 8) appears to correctly account for the main features of the trajectory results. Accordingly, since the noncoupled A-type $\mathrm{HO}_{2}^{*}$ molecules must be associated with specific "inactive" rotational modes, the rate $k_{\mathrm{a}}$ should be inversely related to the amount of energy in those modes, which may increase with initial $E_{\mathrm{r}}$. In fact, we conclude from Figure $3 \mathrm{a}$ and Table 2 that not only does $k_{\mathrm{a}}$ decrease on passing from $E_{\mathrm{r}}=16.980 \mathrm{kcal} \mathrm{mol}^{-1}$ to $E_{\mathrm{r}}=33.961 \mathrm{kcal} \mathrm{mol}^{-1}$ but also the fractions of initially prepared A-type and B-type $\mathrm{HO}_{2}^{*}$ molecules are interchanged, increasing from $f_{\mathrm{A}}^{0}=0.21$ for the former value of the rotational energy to $f_{\mathrm{A}}^{0}=0.72$ for the latter.

In order to associate the noncoupled A-type species with a specific kind of initial preparation of the $\mathrm{HO}_{2}^{*}$ molecules, we have run trajectories for the lower rotational excitation $\left(E_{\mathrm{r}}=\right.$ $6.816 \mathrm{kcal} \mathrm{mol}^{-1}$ ), assigning the orientation of the rotational angular momentum to three mutually perpendicular directions. Thus, as described in section 2 , for each case we have allowed the molecule to rotate around only one of the principal axis of inertia shown in Figure 1, which means that $L_{\mathrm{x}}=L_{\mathrm{y}}=0$ and $L_{\mathrm{z}} \neq 0$ for the rotation in the plane of the nuclei and $L_{\mathrm{y}}=L_{\mathrm{z}}$ $=0$ with $L_{\mathrm{x}} \neq 0$ or $L_{\mathrm{x}}=L_{\mathrm{z}}=0$ with $L_{\mathrm{y}} \neq 0$ for rotations out of that plane. The results are summarized in Table 2 and the decay rates shown in Figure 3 (panels $b$ and $c$ ). Note that the excitation (100) of the $L_{\mathrm{x}}$ component leads to fractions of energy in the products similar to those found for the nonrotating system, presenting for the unimolecular dissociation rate also a RRKMtype behavior as displayed in Figure $3 b$. Furthermore, the corresponding value of $k_{\mathrm{b}}$ is lower than in the nonrotating case, but slightly higher than in the equally distributed rotational energy [denoted by (111)]. This may be rationalized from the 
fact that the (100) rotational excitation is coupled with the reaction path, although the arising centrifugal barrier causes the decrease of $k_{\mathrm{b}}$ from $0.91 \mathrm{ps}^{-1}$ for the nonrotating case to 0.64 $\mathrm{ps}^{-1}$ for the rotating one. Because of this we also find a distribution of energy in the products which only favors slightly rotation in comparison with the nonrotating system.

Figure $3 c$ shows the logarithms of decay rates for the (010) and (001) rotational excitations. We can see now that the results for both cases are well fitted by eq 8 , which means that the two-step mechanism may be used to describe the behavior of the dissociating system. This arises because all rotational energy is associated with the components $L_{y}$ or $L_{z}$ of the angular momentum, which seem to be uncoupled to the reaction path. Note that almost the whole set of prepared $\mathrm{HO}_{2}^{*}$ molecules are in both cases A-type, the corresponding fractions being $90 \%$ and $95 \%$ for the (010) and (001) rotational excitations, respectively. The remaining $10 \%$ or $5 \%$ B-type ones are probably due to some coupling with the reaction path, which occurs for points of phase space corresponding to specific vibrational excitations compatible with the microcanonical normal mode sampling. However, it is worth noting that the decay rates $k_{\mathrm{b}}$ are lower than the corresponding value for the equally excited (111) situation, which suggests a weaker coupling with the reaction path for such $B$-type $\mathrm{HO}_{2}^{*}$ molecules. In fact, the (010) excitation seems to be essentially associated with the bending vibrational motion, which leads to an increase in the number of isomerizations. Moreover, the (010) and (001) preparations give rise to high rotationally excited $\mathrm{O}_{2}$ product molecules, while the corresponding values for $f_{\left\langle E^{\prime}\right\rangle}$ are the lowest ones. Clearly, this suggests that the vibrational-rotational interaction is practically inactive.

As far as the total internal energy increases to $E_{\mathrm{tot}}=76.412$ $\mathrm{kcal} \mathrm{mol}{ }^{-1}$ the reactive $\mathrm{O}+\mathrm{OH}$ channel opens, leading to competitive processes between this channel and the $\mathrm{H}+\mathrm{O}_{2}$ one, which causes additional problems in the interpretation of the results. The trajectory results for formation of $\mathrm{H}+\mathrm{O}_{2}$ and $\mathrm{O}+\mathrm{OH}$ are presented in Tables 3 and 4, respectively. In addition, we show in Figure 4 the corresponding logarithm of the decay rates as a function of time for both dissociative channels. For $\mathrm{H}+\mathrm{O}_{2}$ formation, the fractions $f_{\left\langle E^{\prime}\right\rangle}, f_{\left\langle E^{\prime}\right\rangle}$, and $f_{\langle E\{r\rangle}$, shown in Table 3 present a pattern similar to that found for $E_{\mathrm{tot}}=59.432 \mathrm{kcal} \mathrm{mol}^{-1}$ as the initial rotational energy increases. Also seen from Table 3 is the fact that $k_{\mathrm{b}}$ decreases as the initial rotational excitation increases, the values always being larger than for $E_{\text {tot }}=59.432 \mathrm{kcal} \mathrm{mol}^{-1}$ due to the significant increase in the internal energy. It is possible to observe in Figure 4a that the decay rates present an essentially RRKM behavior even for the highest rotational excitation, which can be explained by the increase in the total energy of the system, clearly above the $\mathrm{H}+\mathrm{O}_{2}$ reaction threshold. However, the (100) excitation leads once again to an increase in the decay rate for the dissociation $\mathrm{HO}_{2}^{*} \rightarrow \mathrm{O}_{2}+\mathrm{H}$, while the $(010)$ and (001) excitations present two distinct decay regimes in Figure $4 \mathrm{~b}$, although not so pronounced as shown before for $E_{\text {tot }}=$ $59.432 \mathrm{kcal} \mathrm{mol}^{-1}$.

In contrast, the number of events leading to the formation of $\mathrm{O}+\mathrm{OH}$ are significant only for the nonrotating system and for $E_{\mathrm{tot}}=16.980 \mathrm{kcal} \mathrm{mol}^{-1}$ with (010) and (001) initial excitations. In the other cases, the number of trajectories forming $\mathrm{O}+\mathrm{OH}$ is too small to get conclusions. This means that rotational degrees of freedom of equally excited molecules (111), but mainly those associated with (100) excitation, tend to be coupled with the reaction path to give $\mathrm{H}+\mathrm{O}_{2}$ products, as it has already been found for $E_{\mathrm{tot}}=59.432 \mathrm{kcal} \mathrm{mol}^{-1}$. Since for nonrotating molecules the "democratically" distributed
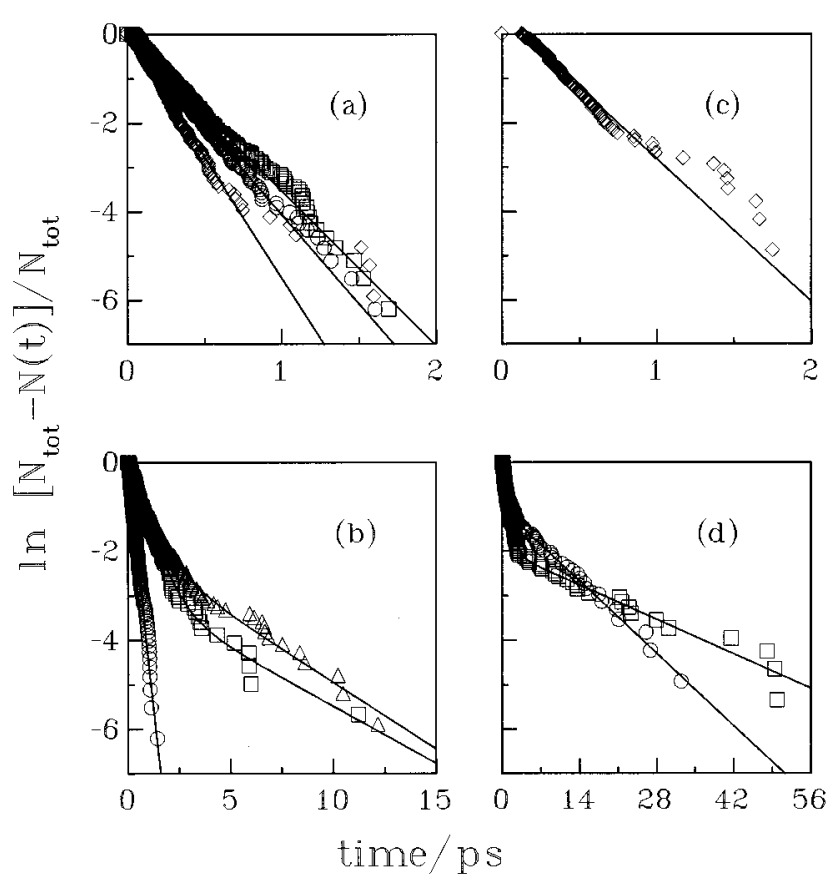

Figure 4. Logarithm of the decay rate for rotating $\mathrm{HO}_{2}^{*}$ molecules as a function of time at $E_{\text {tot }}=76.412 \mathrm{kcal} \mathrm{mol}^{-1}$. Panels a and b are for the $\mathrm{H}+\mathrm{O}_{2}$ products, while panels $\mathrm{c}$ and $\mathrm{d}$ show the results for formation of O + OH. Panel a: $\diamond, E_{\mathrm{r}}=0.0 \mathrm{kcal} \mathrm{mol}^{-1} ; O, E_{\mathrm{r}}=16.980 \mathrm{kcal}$ $\mathrm{mol}^{-1} ; \square, E_{\mathrm{r}}=23.797 \mathrm{kcal} \mathrm{mol}^{-1}$. Panel b: $\bigcirc, E_{\mathrm{r}}=16.980 \mathrm{kcal} \mathrm{mol}^{-1}$ with excitation associated to $L_{x} ; \Delta, L_{y} ; \square, L_{z}$. Panel c: $\diamond, E_{\mathrm{r}}=0.0$ kcal mol${ }^{-1}$. Panel d: $O, E_{\mathrm{r}}=16.980 \mathrm{kcal} \mathrm{mol}^{-1}$ with excitation associated to $L_{y} ; \square, L_{z}$. See text.

vibrational energy is higher than the $\mathrm{O}+\mathrm{OH}$ reactive threshold, the $\mathrm{HO}_{2}^{*}$ species may dissociate to form $\mathrm{OH}$ if the high excited vibrational modes are coupled with the corresponding reaction path, which appears to be the case for 131 trajectories. However, since the "prompt to reaction" molecules are supposed to originate $\mathrm{H}+\mathrm{O}_{2}$ products, dissociation to $\mathrm{O}+\mathrm{OH}$ occurs after a delay of $\sim 0.12 \mathrm{ps}$, followed by a steady rate of 3.22 $\mathrm{ps}^{-1}$, represented by the straight line of Figure 4c. Conversely, for $E_{\mathrm{r}}=16.980 \mathrm{kcal} \mathrm{mol}^{-1}$ with (010) and (001) excitations the vibrational energy is not sufficient to open the $\mathrm{O}+\mathrm{OH}$ channel and some portion of the initial rotational energy has to be used to break the $\mathrm{O}-\mathrm{OH}$ bonds. Although the initial vibrational energy is quite above the $\mathrm{H}+\mathrm{O}_{2}$ reactive threshold, the number of trajectories leading to $\mathrm{O}+\mathrm{OH}$ is significant, which points to the fact that the rotational degrees of freedom associated with the (010) and (001) excitations are preferentialy coupled to the $\mathrm{O}+\mathrm{OH}$ reaction path as might be anticipated from Figure 1. In fact, although a clear two-step mechanism is shown in Figure $4 d$ for the initial (010) and (001) rotational excitations, it is important to note that most $\mathrm{HO}_{2}^{*}$ molecules are prepared as B-type ones in both cases, such molecules being promptly dissociated into $\mathrm{O}+\mathrm{OH}$ products.

\section{Concluding Remarks}

We have shown that nonrotating $\mathrm{HO}_{2}^{*}$ dissociation presents no mode specificity. In fact, even for energies near the $\mathrm{H}+$ $\mathrm{O}_{2}$ threshold, the system shows a clear RRKM-type (i.e., single inverse exponential) behavior. Moreover, a "democratic" excitation of the three vibrational modes allows a better coupling among them, and hence enhances the unimolecular dissociation rate. The fractions of vibrational, rotational, and translational energies in the products have also been found to be nearly constant irrespective of the initial excitation.

As some rotational excitation is added to the molecule, the decaying behavior is no longer RRKM, being now described 
by a double inverse exponential function which indicates the existence of bottlenecks in phase space. Such bottlenecks have been shown to be associated with the rotational excitations (010) and (001) (i.e., around the $L_{y}$ and $L_{z}$ axis, respectively). In fact, these rotational degrees of freedom are associated with total angular momentum ( $\mathbf{J})$ conservation and hence are inactive (i.e., do not readily transfer energy to promote dissociation), while $L_{x}$ is related to the projection of $\mathbf{J}$ onto the body-fixed axis and is not a constant of the motion. Such findings are related to those described in our previous work, ${ }^{17}$ where the preparation of the $\mathrm{HO}_{2}^{*}$ complexes has been done randomly using $\mathrm{H}+\mathrm{ArO}_{2}$ collisions. Indeed, we have then observed that for small collision energies there is a small coupling between rotational and vibrational degrees of freedom so that the complexes require an induction time prior to dissociation.

We have also studied rotational effects for a total internal energy above the threshold for $\mathrm{O}+\mathrm{OH}$ dissociation. In this case, there is a competition between the $\mathrm{H}+\mathrm{O}_{2}$ and $\mathrm{O}+\mathrm{OH}$ reactive events, although the former is clearly favored. Indeed, for most sets of initial conditions that include molecular rotation, only a few trajectories led to $\mathrm{OH}$ formation. The exceptions to this trend arise for the (010) and (001) rotational excitations where a large number of dissociations occurs to form $\mathrm{OH}$. This kind of excitation, less coupled with the reaction path for $\mathrm{O}_{2}$ formation, favors $\mathrm{O}+\mathrm{OH}$ dissociation, especially for trajectories leading to a large number of isomerizations.

In summary, rotational effects (including the initial $\mathbf{J}$ orientation) have shown to be important in characterizing the dissociation of $\mathrm{HO}_{2}^{*}$, even for internal energies above the $\mathrm{O}+\mathrm{OH}$ threshold. Since quantum dynamical calculations on the $\mathrm{HO}_{2}$ system are currently affordable only for $\mathbf{J}=0$, they cannot be used to account for such rotational effects (see also ref 25). Thus, trajectory calculations are the only available approach to fully describe the phenomena associated with $\mathrm{HO}_{2}^{*}$ dissociation.

Acknowledgment. The financial support from Junta Nacional de Investigação Científica e Tecnológica (JNICT), Portugal, is gratefully acknowledged.

\section{References and Notes}

(1) Steinfeld, J. I.; Francisco, J. S.; Hase, W. L. Chemical Kinetics and Dynamics; Prentice Hall: Englewood Cliffs, NJ, 1989. 704.

(2) Morais, V. M. F.; Varandas, A. J. C. J. Phys. Chem. 1992, 96,

(3) Marcus, R. A. J. Chem. Phys. 1952, 20, 359.

(4) Marcus, R. A.; Rice, O. K. J. Phys. Colloid Chem. 1951, 55, 894.

(5) Guan, Y.; Lynch, G. C.; Thompson, D. L. J. Chem. Phys. 1987, 87, 6957.

(6) Preiskorn, A.; Thompson, D. L. J. Chem. Phys. 1989, 91, 2299.

(7) Guan, Y.; Thompson, D. L. Chem. Phys. 1989, 139, 147.

(8) Marks, A. J. J. Chem. Phys. 1994, 100, 8096.

(9) Miller, J. A.; Brown, N. J. J. Phys. Chem. 1982, 86, 772.

(10) Melius, C. F.; Blint, R. J. Chem. Phys. Lett. 1979, 64, 183.

(11) Pastrana, M. R.; Quintales, L. A. M.; Brandão, J.; Varandas, A. J. C. J. Phys. Chem. 1990, 94, 8073.

(12) Mandelshtam, V. A.; Grozdanov, T. P.; Taylor, H. S. J. Chem. Phys. 1995, 103, 10074.

(13) Mahapatra, S. J. Chem. Phys. 1996, 105, 344.

(14) Dobbyn, A. J.; Stumpf, M.; Keller, H.-M.; Hase, W. L.; Shinke, R. J. Chem. Phys. 1995, 103, 9947.

(15) Dobbyn, A. J.; Stumpf, M.; Keller, H.-M.; Schinke, R. J. Chem. Phys. 1996, 104, 8357.

(16) Song, K.; Peslherbe, G. H.; Hase, W. L. J. Chem. Phys. 1995, 103, 8891.

(17) Marques, J. M. C.; Wang, W.; Pais, A. A. C. C.; Varandas, A. J. C. J. Phys. Chem. 1996, 100, 17513.

(18) Varandas, A. J. C.; Brandão, J.; Pastrana, M. R. J. Chem. Phys. 1992, 96, 5137

(19) Varandas, A. J. C.; Marques, J. M. C. J. Chem. Phys. 1992, 97, 4050 .

(20) Varandas, A. J. C. J. Chem. Phys. 1993, 99, 1076.

(21) Varandas, A. J. C. Chem. Phys. Lett. 1994, 225, 18.

(22) Varandas, A. J. C.; Marques, J. M. C. J. Chem. Phys. 1994, 100, 1908.

(23) Marques, J. M. C.; Wang, W.; Varandas, A. J. C. J. Chem. Soc., Faraday Trans. 1994, 90, 2189.

(24) Varandas, A. J. C. Chem. Phys. Lett. 1995, 235, 111.

(25) Varandas, A. J. C. Mol. Phys. 1995, 85, 1159.

(26) Varandas, A. J. C.; Pais, A. A. C. C.; Marques, J. M. C.; Wang, W. Chem. Phys. Lett. 1996, 249, 264.

(27) Hase, W. L. MERCURY: a general Monte-Carlo classical trajectory computer program, QCPE No. 453. An updated version of this program is VENUS96: Hase, W. L.; Duchovic, R. J.; Hu, X.; Komornick, A.; Lim, K. F.; Lu, D.-H.; Peslherbe, G. H.; Swamy, K. N.; Van de Linde, S. R.; Varandas, A. J. C.; Wang, H.; Wolf, R. J. QCPE Bull. 1996, 16, 43.

(28) Bintz, K. L.; Thompson, D. L. J. Chem. Phys. 1986, 85, 1848.

(29) Raff, L. M.; Thompson, D. L. Theory of Chemical Reaction Dynamics, M. Baer, Ed.; Chemical Rubber Co.: Boca Raton; FL, 1985; p 1 .

(30) Sloane, C. S.; Hase, W. L. J. Chem. Phys. 1977, 66, 1523. 